\title{
Tocilizumab and Thromboembolism in COVID-19: A Retrospective Hospital-Based Cohort Analysis
}

Kok Hoe Chan ${ }^{1}$, Bhavik Patel ${ }^{2}$, Bishnu Podel ${ }^{2}$, Maria E. Szablea ${ }^{3}$, Hamid S. Shaaban ${ }^{4}$, Gunwant Guron ${ }^{4}$, Jihad Slim ${ }^{3}$

1. Internal Medicine, Saint Michael's Medical Center, Newark, USA 2. Medical Education, Saint Michael's Medical Center, Newark, USA 3. Infectious Diseases, Saint Michael's Medical Center, Newark, USA 4. Hematology and Oncology, Saint Michael's Medical Center, Newark, USA

Corresponding author: Kok Hoe Chan, kokhoedelcos.chan@gmail.com

\section{Abstract \\ Background}

Tocilizumab, an interleukin-6 (IL-6) receptor antagonist, has been used in patients with coronavirus disease 2019 (COVID-19) as an anti-cytokine agent. IL-6 also plays a complex role in hemostasis and thrombosis. We observed a transient elevation of D-dimer in our patients who received tocilizumab, which triggered this study.

\section{Methods}

A retrospective hospital-based cohort analysis of patients with confirmed COVID-19 who received tocilizumab during the study period of March 15, 2020, to May 20, 2020, was conducted. We retrieved demographic, clinical, and laboratory data, and patients who were receiving therapeutic anticoagulation therapy prior to tocilizumab administration were excluded. Descriptive analysis was performed, and the cause of death and trends of D-dimer and inflammatory markers were studied.

\section{Results}

Out of the 436 confirmed COVID-19 patients admitted during the study period, 24 met the inclusion criteria. Their median age was 47.5 years. They were 18 males and 6 females; 15 patients survived and nine expired. Of the group that survived, 12 received therapeutic anticoagulation. Of the seven patients who did not receive therapeutic anticoagulation, four expired (one from sepsis and three probably from thromboembolic complications) compared to five deaths in the 17 patients who received therapeutic anticoagulation (four from sepsis and one possibly from thromboembolic complications).

\section{Conclusions}

The interplay between IL-6, IL-6 receptor antagonist, and venous thromboembolism is complex. We observed a transient elevation of D-dimer in COVID-19 patients who received tocilizumab, and a trend toward increased death secondary to thromboembolism. This observation is novel and highlights the

Review began 05/17/2021 Review ended 05/18/2021 Published 05/24/2021

\section{() Copyright 2021}

Chan et al. This is an open access article distributed under the terms of the Creative Commons Attribution License CC-BY 4.0., which permits unrestricted use, distribution, and reproduction in any medium, provided the original author and source are credited. potential thrombophilic side effects of tocilizumab.

Categories: Internal Medicine, Infectious Disease, Hematology

Keywords: coronavirus 2019, sars-cov-2, tocilizumab, il-6, hypercoagulable state, thrombosis, il-6 receptor antagonist

\section{Introduction}

Coronavirus disease 2019 (COVID-19) is a major public health emergency. COVID-19 has multifaceted presentations, and while the majority of patients are asymptomatic or present with mild disease, there is a subgroup of patients who tend to present with severe disease. Cytokine storm mediated by proinflammatory cytokines such as interleukin-6 (IL-6) and tumor necrosis factor-alpha (TNF- $\alpha$ ) is probably the culprit for severe COVID-19 [1]. Exuberant inflammatory response with complications related to cytokine release syndrome have been observed and proposed as leading to critical and fatal illness $[2,3]$.

Currently, there are two recombinant humanized monoclonal antibodies targeting the IL- 6 receptor that are approved by the FDA in the USA: sirukumab and tocilizumab. There are multiple case reports and observational studies reporting the use of tocilizumab in patients with COVID-19 from around the world [46]. In the observational study by Xu et al., 21 patients with severe or critical COVID-19 infections showed rapid fever reduction and reduced oxygen requirement after receiving tocilizumab [4], highlighting the potential role of IL-6 in modulating the disease activity.

To the best of our knowledge, tocilizumab has not been shown to increase the risk of thrombosis or 
hypercoagulable state. Nonetheless, we have observed an elevation of D-dimer in the patients receiving tocilizumab while the rest of the inflammatory markers (ferritin, lactate dehydrogenase [LDH], and Creactive protein $[\mathrm{CRP}]$ ) were down-trending, with an increased mortality related to thromboembolism, raising the possibility that tocilizumab may be promoting a prothrombic state. In this retrospective hospitalbased cohort study, we report the clinical outcomes and dissect the potential interplay between tocilizumab and thrombosis.

This article was previously posted to the Research Square preprint server on July 16, 2020.

\section{Materials And Methods}

A retrospective analysis was conducted of patients admitted to our hospital between March 15, 2020, and May 20, 2020. Inclusion criteria were as follows: (1) positive SARS-CoV-2 RT-PCR (severe acute respiratory syndrome coronavirus 2 reverse transcription polymerase chain reaction) and (2) received tocilizumab as part of their COVID-19 regimen. The only exclusion criterion was prior anticoagulation use.

Demographic analysis was performed, and data were expressed as counts, percentages, or median. Death was determined as thromboembolic disease if the patient expired with increasing D-dimer, while ferritin and CRP were decreasing and as septic shock if cultures were positive, increasing vasopressor requirement and/or increasing CRP and/or ferritin. Death was unlikely due to thromboembolic if D-dimer was $<1,500$ at the time of death. The trends of D-dimer and inflammatory markers of the patients who did not receive anticoagulation are plotted and shown in Figure 1. A waiver of HIPAA (Health Insurance Portability and Accountability Act) privacy authorization was been obtained through the hospital Local Institutional Review board.

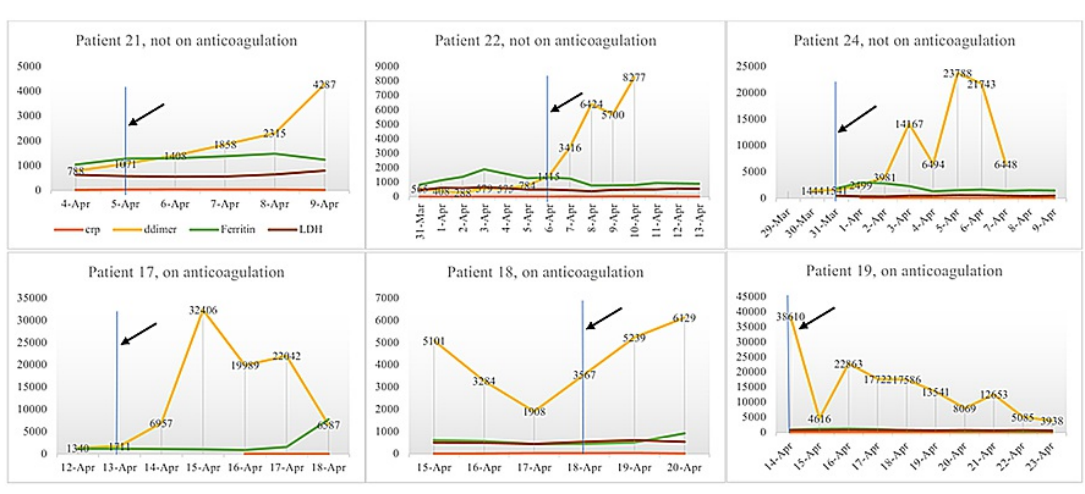

\section{FIGURE 1: Trend of D-dimers and inflammatory markers (CRP, LDH, and ferritin) of the patients who expired.}

Blue line and black arrow indicate the time of the tocilizumab given, orange line indicates CRP, yellow line indicates D-dimer, green line indicates ferritin, and brown line indicates LDH.

CRP, C-reactive protein; LDH, lactate dehydrogenase

\section{Results}

Between March 15, 2020, and May 20, 2020, 24 SARS-CoV-2 RT-PCR tested positive patients received one dose of tocilizumab $400 \mathrm{mg}$ IV. Patients' demographics and outcomes are tabulated in Table 1. 


\section{Cureus}

\begin{tabular}{|c|c|c|c|c|c|c|c|c|}
\hline No. & $\begin{array}{l}\text { Age, } \\
\text { years }\end{array}$ & Gender & Race & $\begin{array}{l}\text { No. of days } \\
\text { of } \\
\text { symptoms }\end{array}$ & $\begin{array}{l}\text { No. of days from } \\
\text { symptoms to } \\
\text { tocilizumab }\end{array}$ & $\begin{array}{l}\text { Therapeutic } \\
\text { anticoagulation }\end{array}$ & Outcome & Cause of death \\
\hline 1 & 38 & Male & Hispanic & 7 & 11 & Yes & Survived & - \\
\hline 2 & 41 & Male & Hispanic & 3 & 3 & Yes & Survived & - \\
\hline 3 & 43 & Female & Hispanic & 9 & 11 & Yes & Survived & - \\
\hline 4 & 44 & Male & Other & 10 & 10 & Yes & Survived & - \\
\hline 5 & 44 & Male & Hispanic & 7 & 11 & Yes & Survived & - \\
\hline 6 & 46 & Male & Hispanic & 7 & 9 & Yes & Survived & - \\
\hline 7 & 50 & Male & Other & 14 & 19 & Yes & Survived & - \\
\hline 8 & 57 & Male & Hispanic & 14 & 16 & Yes & Survived & - \\
\hline 9 & 60 & Female & AA & 7 & 10 & Yes & Survived & - \\
\hline 10 & 60 & Female & Hispanic & 7 & 10 & Yes & Survived & - \\
\hline 11 & 61 & Female & Other & 14 & 15 & Yes & Survived & - \\
\hline 12 & 67 & Male & AA & 5 & 10 & Yes & Survived & - \\
\hline 13 & 34 & Male & Hispanic & 7 & 10 & No & Survived & - \\
\hline 14 & 44 & Female & Other & 3 & 3 & No & Survived & - \\
\hline 15 & 60 & Female & Hispanic & 7 & 7 & No & Survived & - \\
\hline 16 & 37 & Male & Hispanic & 2 & 3 & Yes & Expired & $\begin{array}{l}\text { Septic shock with acute rise of } \\
\text { CRP/ferritin }\end{array}$ \\
\hline 17 & 42 & Male & Hispanic & 5 & 9 & Yes & Expired & $\begin{array}{l}\text { AHRF likely due to thromboembolic } \\
\text { event }\end{array}$ \\
\hline 18 & 49 & Male & Hispanic & 7 & 8 & Yes & Expired & $\begin{array}{l}\text { Septic shock and multiorgan fallure } \\
\text { increasing pressor requirement (three } \\
\text { pressors) }\end{array}$ \\
\hline 19 & 52 & Male & Hispanic & 7 & 9 & Yes & Expired & $\begin{array}{l}\text { Septic shock (blood culture positive } \\
\text { for Neisseria sicca) }\end{array}$ \\
\hline 20 & 62 & Male & ispanic & 7 & 14 & Yes & Expired & $\begin{array}{l}\text { Septic shock with increasing } \\
\text { CRP/ferritin }\end{array}$ \\
\hline 21 & 35 & Male & Hispanic & 14 & 18 & No & Expired & $\begin{array}{l}\text { AHRF likely due to thromboembolic } \\
\text { event }\end{array}$ \\
\hline 22 & 39 & Male & Hispanic & 7 & 8 & No & Expired & $\begin{array}{l}\text { AHRF likely due to thromboembolic } \\
\text { event }\end{array}$ \\
\hline 23 & 52 & Male & Hispanic & 5 & 7 & No & Expired & $\begin{array}{l}\text { Septic shock and severe Clostridiodes } \\
\text { difficile }\end{array}$ \\
\hline 24 & 60 & Male & Hispanic & 7 & 10 & No & xpired & $\begin{array}{l}\text { AHRF likely due to thromboembolic } \\
\text { event }\end{array}$ \\
\hline
\end{tabular}

TABLE 1: Demographic, clinical outcomes, and cause of death after tocilizumab.

AA, African American; AHRF, acute hypoxic respiratory failure; CRP, C-reactive protein

Their median age was 47.5 years (range: 32-67 years). They were 18 males and 6 females. As for ethnicity, 17 (74\%) were Hispanic, two (9\%) were African American, and four (17\%) belonged to other ethnicities. Of the 24 patients, 15 (65\%) patients survived and nine (35\%) patients expired. Therapeutic anticoagulation with either low molecular weight heparin or unfractionated heparin was given to 17 (71\%) patients. Of the 15 
patients who survived, 12 (80\%) patients received therapeutic anticoagulation. Only five (56\%) patients in the expired group received therapeutic anticoagulation. Four patients who were not anticoagulated expired (one from sepsis and three probably from thromboembolic complications) compared to five deaths in the 17 patients who received therapeutic anticoagulation (four from sepsis and one possibly from thromboembolic complications).

Out of 24 patients, 18 (75\%) had elevation of D-dimer after tocilizumab administration, while all the inflammatory markers (ferritin, $\mathrm{LDH}$, and CRP) were trending down. Moreover, the elevation was more pronounced in the group of patients who expired and not on therapeutic anticoagulation (Figure 1).

\section{Discussion}

The association between tocilizumab and thrombosis, to our knowledge, has never been reported. We observed an elevation of D-dimer after tocilizumab administration. Moreover, in four patients who were not anticoagulated and expired, there was a profound elevation of D-dimer, and three of them expired probably secondary to thromboembolic events. As compared to those who were anticoagulated, most of them expired due to sepsis and only one expired likely secondary to thromboembolic event.

The interplay between IL-6, IL-6 receptor antagonist, and venous thromboembolism is complex. IL-6 is a proinflammatory cytokine that is involved in inflammation, autoantibody production, endothelial regeneration, and permeability, as well as hematopoiesis [7]. IL-6 is produced by a variety of cells including T-lymphocytes, monocytes, endothelial cells, and fibroblasts [7]. Stone et al. has demonstrated an increased risk of deep vein thrombosis with tumor-derived IL-6 in a mouse model of ovarian cancer via the induction of hepatic thrombopoietin, promoting thrombus formation [8]. Moreover, IL- 6 has been reported to contribute to deep vein thrombosis by dysregulation of miR-338-5p expression [9].

On the contrary, Nosaka et al. has recently published a research article highlighting the role of IL-6 in thrombus resolution, in which suppression of IL-6 may, in fact, result in the growth of the thrombus, as demonstrated in the rat model [10]. Moreover, IL-6 antagonist has been associated with a reduced level of factor XIII, chimerin, and plasminogen activator inhibitor [11]. The pivotal role of factor XIII is in fibrin stabilization, thus the decrease of factor XIII as a result of IL-6 antagonist will lead to thrombus instability, which may also contribute to this thrombophilic state.

Thrombosis is a complex phenomenon, and the hemostasis balance between thrombus formation rate and resolution is important. IL-6 in this context seems to play a complex role in both thrombus formation and resolution. This assumption has been strengthened by the observations that the administration of tocilizumab, an IL-6 antagonist, transiently elevated the D-dimer, which is a marker of coagulability, though more studies are needed to understand the pathophysiological role of IL-6 in hemostasis and thrombosis. Our observation suggests that it may be beneficial to use therapeutic anticoagulation in COVID 19 patients receiving tocilizumab, as they already are at high risk of thrombosis.

\section{Conclusions}

The interplay between IL-6, IL-6 receptor antagonist, and venous thromboembolism is complex. We observed a transient elevation of D-dimer in COVID-19 patients who received tocilizumab. This observation is novel and highlights the potential thrombophilic side effects of tocilizumab. Prospective randomized controlled trials will need to measure this effect in order to come up with a firm clinical recommendation in the future.

\section{Additional Information \\ Disclosures}

Human subjects: Consent was obtained or waived by all participants in this study. Animal subjects: All authors have confirmed that this study did not involve animal subjects or tissue. Conflicts of interest: In compliance with the ICMJE uniform disclosure form, all authors declare the following: Payment/services info: All authors have declared that no financial support was received from any organization for the submitted work. Financial relationships: All authors have declared that they have no financial relationships at present or within the previous three years with any organizations that might have an interest in the submitted work. Other relationships: All authors have declared that there are no other relationships or activities that could appear to have influenced the submitted work.

\section{References}

1. Siddiqi HK, Mehra MR: COVID-19 illness in native and immunosuppressed states: a clinical-therapeutic staging proposal. J Heart Lung Transplant. 2020, 39:405-7. 10.1016/j.healun.2020.03.012

2. Huang C, Wang Y, Li X, et al.: Clinical features of patients infected with 2019 novel coronavirus in Wuhan, China. Lancet. 2020, 395:497-506. 10.1016/S0140-6736(20)30183-5

3. Mehta P, McAuley DF, Brown M, Sanchez E, Tattersall RS, Manson JJ: COVID-19: consider cytokine storm syndromes and immunosuppression. Lancet. 2020, 395:1033-4. 10.1016/S0140-6736(20)30628-0 


\section{Cureus}

4. Xu X, Han M, Li T, et al.: Effective treatment of severe COVID-19 patients with tocilizumab . Proc Natl Acad Sci U S A. 2020, 117:10970-5. 10.1073/pnas.2005615117

5. Luo P, Liu Y, Qiu L, Liu X, Liu D, Li J: Tocilizumab treatment in COVID-19: a single center experience . J Med Virol. 2020, 92:814-8. 10.1002/jmv.25801

6. Alberici F, Delbarba E, Manenti C, et al.: A single center observational study of the clinical characteristics and short-term outcome of 20 kidney transplant patients admitted for SARS-CoV2 pneumonia. Kidney Int. 2020, 97:1083-8. 10.1016/j.kint.2020.04.002

7. Murakami M, Kamimura D, Hirano T: Pleiotropy and specificity: insights from the interleukin 6 family of cytokines. Immunity. 2019, 50:812-31. 10.1016/j.immuni.2019.03.027

8. Stone RL, Nick AM, McNeish IA, et al.: Paraneoplastic thrombocytosis in ovarian cancer. N Engl J Med. 2012, 366:610-8. 10.1056/NEJMoa1110352

9. Zhang Y, Zhang Z, Wei R, et al.: IL (interleukin)-6 contributes to deep vein thrombosis and is negatively regulated by miR-338-5p. Arterioscler Thromb Vasc Biol. 2020, 40:323-34. 10.1161/ATVBAHA.119.313137

10. Nosaka M, Ishida Y, Kimura A, et al.: Crucial involvement of IL-6 in thrombus resolution in mice via macrophage recruitment and the induction of proteolytic enzymes. Front Immunol. 2019, 10:3150. 10.3389/fimmu.2019.03150

11. Jewell P, Ansorge O, Kuker W, Irani SR, Zamboni G: Tocilizumab-associated multifocal cerebral thrombotic microangiopathy. Neurol Clin Pract. 2016, 6:e24-6. 10.1212/CPJ.0000000000000220 